\title{
Simulation of Biological Ionic Channels by Technology Computer-Aided Design
}

\author{
K. HESS ${ }^{\mathrm{a}}$, U. RAVAIOLI ${ }^{\mathrm{a} *}$, M. GUPTA ${ }^{\mathrm{a}}$, N. ALURU ${ }^{\mathrm{a}}$, \\ T. VAN DER STRAATEN ${ }^{b}$ and R. S. EISENBERG ${ }^{b}$

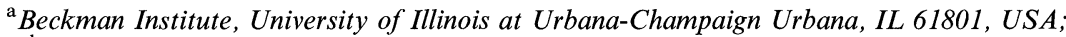 \\ ${ }^{\mathrm{b}}$ Department of Molecular Biophysics, Rush Medical Center, Chicago, IL 60612, USA
}

\begin{abstract}
This paper discusses the use of established Technology Computer-Aided Design (TCAD) tools and methodologies for the study of charge transport in molecular biology systems, like ionic channels, that display a behavior analogous to electronic devices. Continuum drift-diffusion and Monte Carlo methods can be applied to analyze steadystate and transient behavior of ionic channels over time scales that cannot be resolved practically by detailed molecular dynamics or quantum approaches. The difficult ionwater interaction can be lumped phenomenologically into mobility or scattering rate parameters, while the solution of Poisson equation over the complete domain provides a simple way to include external boundary conditions and image force effects at dielectric discontinuities. We present here some recent results of 3-D simulations for a gramicidin ion channel, obtained using the rapid prototyping computational platform PROPHET.
\end{abstract}

Keywords: Ionic channels; Transport simulation; Nanotechnology

\section{INTRODUCTION}

Ionic channels are naturally occurring nanotubes that control the movement of ions in and out of cells, providing communication through the insulating cell membrane [1-3]. Ionic channels are a class of proteins consisting of a few hundred to a few thousand atoms, with strong and rapidly varying localized charge. These bio-molecular structures have very intersting similarities with nanoscale solid-state structures and have the potential to be employed in a range of nanotechnology applications. There are many types of channels with very specialized behavior, often resembling the functionality of complex electronics systems. Some channels exhibit strong selectivity, having the ability to let only certain types of ions traverse the pore, other channels can shut off the ion flow under the influence of external electrical or mechanical action. The possibility to incorporate such bio-molecular structures in electronics circuits as sensing, memory, or even

*Address for correspondence: 3255 Beckman Institute, University of Illinois, 405 N. Mathews Avenue, Urbana IL 61801, USA. Tel.: +1 217244 5765, Fax: +9144811 1955, 4901, e-mail: ravaioli@uiuc.edu 
computational elements, opens up exciting opportunities and great challenges. When the channel pore is open, ions move essentially by a driftdiffusion process, but from the computational point of view, detailed simulation of ionic channels is a formidable problem, because of the strong coulomb interaction with the fixed charges and because of the interaction between ions and water molecules. While complete molecular dynamics calculations have been attempted [4], these can only resolve very short time scales, many orders of magnitude smaller than the times involved with the biological functions performed by the channels. Even with fairly high concentrations of salts (e.g., $\mathrm{KCl}, \mathrm{NaCl}$ ) one has to include tens or hundreds of thousands water molecules per ion. At the same, simulations must include a realistic description of transmembrane potential and electric field, because they are involved in every function of the channels. Measurements of ion channel conductivity are made by applying a bias with fine needle-like electrodes, but in general it is difficult to recreate these conditions within a molecular dynamics simulation. This happens partly because out-of-equilibrium conditions generated by the external potential are not easily included in a molecular dynamics algorithm and partly because the dielectric constants of the water solution, of the protein lining the pore, and of the lipids surrounding the protein vary greatly. Rapid variations of the dielectric constants distort the electric fields on a nanometer scale. This would require the resolution of appropriate image forces in the dielectrics layers, represented by protein and lipids, which is one of the most challenging issues in direct force evaluation by molecular dynamics techniques.

To investigate the macroscopic properties of ionic channels and to address possible applications for device functions, there is the need for a hierarchy of simulation tools, much in the same way as it has been developed for solid-state integrated circuits. It is useful to simulate both the steady-state behavior of the channel and the transient regime over millisecond scale, under conditions very far from equilibrium. In general, one needs to investigate whether the channel is electrostatically transparent and include the long range Coulomb forces of all charges involved. Simulations must also include large gradients of electrical potential and of ion concentration, and must be able to predict large currents because the manipulation of these gradients and measurement of these currents are the customary tools of biophysical investigations. Simulations cannot be done just at equilibrium if biological experiments are to be predicted [5].

\section{SOLID-STATE DEVICES VS. IONIC CHANNELS}

As mentioned above, the modality of operation of ionic channels may resemble the functions performed by electronic devices. However, there are many differences that should be clearly understood when attempting the electrical simulation of ionic channels. Solid-state structures are based on crystalline semiconductor substrates characterized by a band structure which is known for all the important materials. The existence of a welldefined band structure for solids with crystalline materials has been the key to the rapid development of the theory of semiconductor devices [6]. In contrast, transport in ionic channel takes place in the water solution that permeates the membranes. The theory of liquids is much less developed, even if liquid and solid matter have comparable densities, because molecules and ions in the liquid are spatially disordered and not arranged periodically. The analytic framework for transport in liquids is enormously more complicated. In solidstate, the most important features of the crystal matrix are summarized in the effective mass associated to the simulated charge carriers, which are effective quasi-particles, not the real ones. Carriers in water solutions are the actual ions, with a relatively large mass when compared to the effective mass of electrons and holes in semiconductors. 
Conduction channels in solid-state devices are usually delimited by potential variations induced by depletion layers or by potential energy steps at hetero-junctions. Ionic channels are instead delimited by a membrane with high concentration of charge, which varies quite rapidly between neighboring atomic clusters. Coulomb interactions tend to be much stronger in ionic channels. The ions in the solution behave essentially like a plasma, but with similar mass for the species of opposite polarity and no fixed doping charge that in a device generates screening effects mitigating potential fluctuations. When ionic species are separated by selectivity effects, the interaction, between ions in the channel and charges lining the pore, is essentially unscreened. Charge can then pile up in localized regions, with effective density much higher than the one in the solution.

One major problem in solid-state is that fluctuations of dopings and sizes strongly affect devices at the nano-scale. Statistically one has to expect a large number of defects and deviations from specified dimensions and doping profiles in large arrays of devices. Molecular structures are instead always perfect replicas, and it is possible to create stable mutations, also very repeatable, to modify in a predictable way the channel behavior. Crystalline structures are also very susceptible to damage due to energetic carriers, while molecular structures appear to be more resilient.

\section{SIMULATION APPROACHES}

In treating transport in ionic channels from a device viewpoint, the lowest level of the simulation hierarchy is a continuum transport model, equivalent to the drift-diffusion approach [7] in TCAD. The dynamics is treated as a succession of ion flow configurations described as a fluid, where the forces are electrostatically determined. The water is treated as a uniform background medium with a specific dielectric constant. The macroscopic current flow is resolved by assigning an appropriate mobility/diffusivity to the ionic species that must include all the physical effects affecting transport, like the average effective viscosity of the water. The mobilities must also be calibrated further, if one want to describe phenomena like selectivity, by reducing the flow rate of ions that cannot permeate the channel, since a simple flow model cannot by itself resolve the detailed interaction leading to selectivity.

Standard non-linear iteration approaches used for solid state devices are applicable to solve the system of equations for the flow in ionic channel which include Poisson equation and the continuity equations for cations and anions. Within the limitations of the physical model, the strength of the drift-diffusion TCAD approaches is in the capability to handle both local charge interaction and external bias effects via Poisson equation, with an automatic resolution of discontinuities in the dielectric constant.

One needs to understand that the quantities obtained from a drift-diffusion solution represent steady-state averages for an equivalent fluid that attempt to describe an otherwise granular process of ion current. The solution baths at the ends of the channel behave like contacts that continuously inject particles in the mouths of the pore. Only few ions that attempt to enter the channel are able to traverse it. The overall current is the result of a series of rare ionic traversal events, and few ions are inside the channel at any one time. A driftdiffusion approach is however very valuable to analyze global channel behavior. This is very important if one wants to consider extremely large arrays of biomolecular elements for device applications. Even compact models as used in VLSI design will be needed, and drift-diffusion is the first necessary step to understand how to construct such compact models.

A flexible continuum approach can go a long way towards a quantitative description. Besides the aforementioned ability to treat the image force and electrostatic boundary conditions, one could also account for chemical reactions such as binding of an ion, by adding to the continuity equations an effective generation-recombination 
term $U$ that removes ions from the mobile population and adds them to the fixed charges and vice versa. For positively charged ions with elementary charge $q$ the continuity equation reads:

$$
\nabla \cdot \boldsymbol{J}+q(\partial p / \partial t)=-q U
$$

The function $U$ can be of any form that describes the experimental facts of ion binding and localization, or ion delocalization and release. One may be able to describe it in simple cases by a lifetime $\tau$ of the mobile ion concentration $p$, by using an average mobile ion concentration $p_{\mathrm{o}}$ :

$$
U \approx\left(p-p_{o}\right) / \tau
$$

This formulation, available in all TCAD tools, appears to be a suitable way to describe effects of binding, for example at constrictions, and it may also suggest alternative ways to include ion selectivity locally in the continuum picture, although for this new ideas are still needed.

The next interesting level in the hierarchy is particle Monte Carlo simulation [8], which is able to capture in a natural way the discrete features of ion transport inside the channel. The standard approach used for Monte Carlo device simulation can be a good starting point, but a number of changes are necessary. The electrostatic interaction between fixed charges on the channel walls and the channel ions is very strong. In this context of individual particle simulation, Poisson equation, that resolves the long-range interaction, is by itself not sufficient. Individual coulomb forces must be added as done recently for 3-D device simulation, by providing a short-range correction to Poisson equation. This involves an evaluation of local molecular dynamics forces, which then are added to the long-range Poisson mesh force taking care of eliminating double counting in the overlap region between the two domains $[9,10]$. In a simpler approach, only the transport inside the channel is resolved and the contact baths are included as quasi-equilibrium injecting boundary conditions placed close to the channel mouths. In this approach it is practical to split the coulomb forces between ions and fixed charges from external bias effects, which may be included at first order as the potential drop obtained for the channel empty of charges. Coulomb forces due to wall charges can be pre-calculated on a very fine mesh and interpolated in the channel using tables. Only ion-ion forces need to be updated, since few ions are inside the channel at any time step. The cost per time step tends to be small, and the typical time steps necessary to resolve the coulomb interaction are comparable to the ones used in solid-state simulation, on the femtosecond scale. However, conduction averages in an ionic channel are meaningful over much long longer time scales, up to milliseconds. Therefore, the particle solution of ionic channels is a time-multiscale problem that requires very large computational resources. Ionwater interaction is handled also in this case as a viscosity, implemented in the Monte Carlo model by using a uniform scattering rate calibrated from measurement data. This simple model corresponds to imposing a given mobility to the fluid described by drift-diffusion. More attention must be paid in the future to improve the scattering model, which will require a combination of higher level simulations [11] and transport measurements. Also, we have assumed point-like particles in the initial implementation. The next necessary step in improving the model will be to associate a finite size to the ions.

In Figure 1 we give a result from Monte Carlo simulation of sodium ions in gramicidin, a widely studied ionic channel, with a solution concentration of 1.0 molar and applied bias of $100 \mathrm{mV}$. The graph shows statistics of particles as a function of time spent inside the channel. Note that nearly all of the ions represented by this plot did not traverse the channel but were reflected back to the injection contacts. In fact, most of the successful attempts recorded by the simulation, carried over microseconds, occurred for traversal times that could not be accommodated by the plot and are off the time scale. This gives an idea of the rarity of traversal events in these nano-scale channels. 


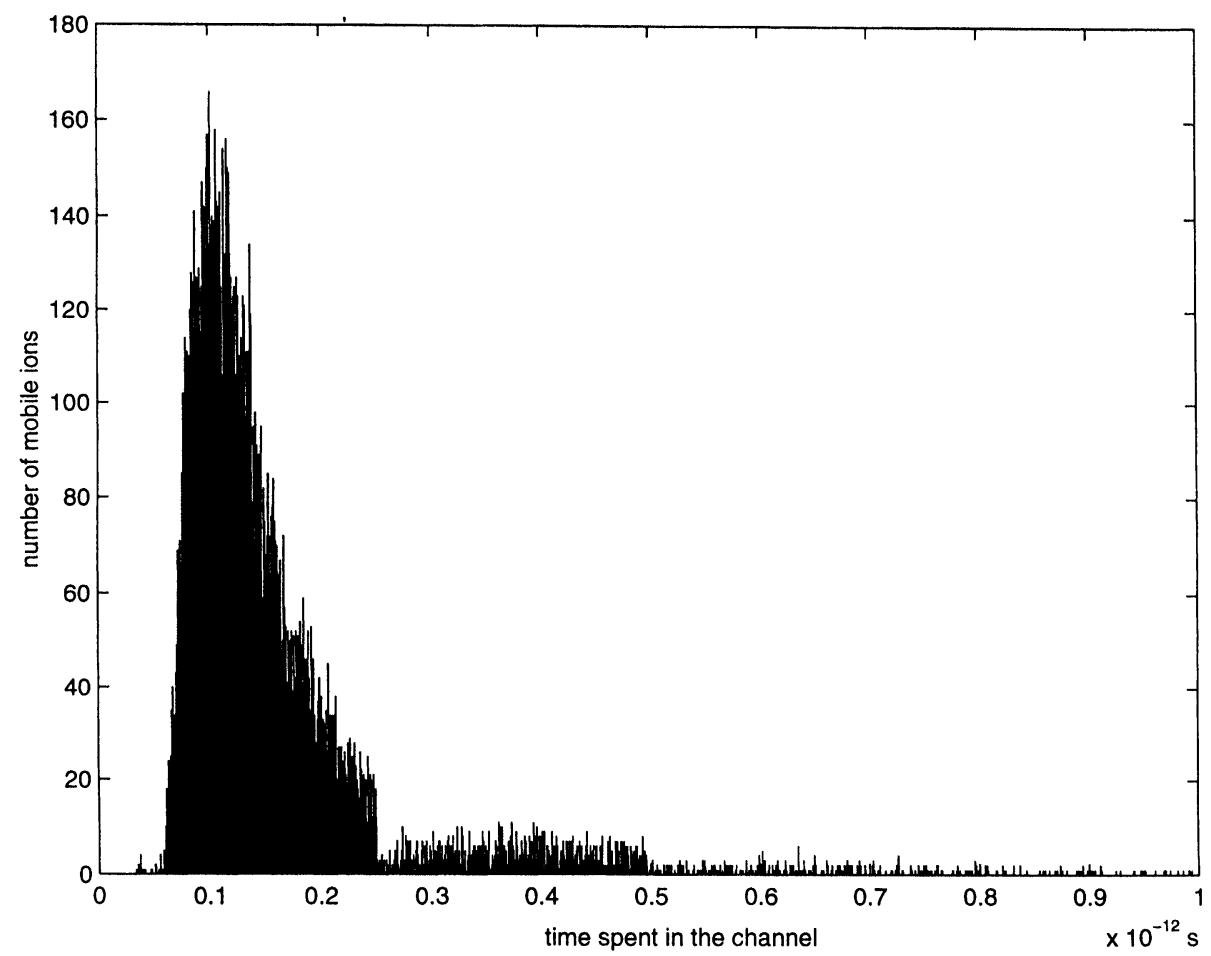

FIGURE 1 Histogram of lingering times for $\mathrm{Na}^{+}$ions in a gramicidin channel, obtain for a solution concentration of 1.0 molar and applied bias $100 \mathrm{mV}$.

\section{3-D CONTINUUM SIMULATIONS}

We limit here the presentation of our results to recent work in multi-dimensional drift-diffusion level simulation, due to space limitations. Earlier, we conducted simulations with a commercial software package, ISE DESSIS [12], to asses the capabilities of standard TCAD tools. We found that it is possible to simulate directly 3-D ionic channel structures if a set of scaling rules are followed, that transform the biological transport problem into an equivalent semiconductor problems, where electrons and holes represent anions and cations, respectively.

Recently, we have initiated the development of customized applications using PROPHET, a rapid prototyping TCAD computational platform originally developed by C. Rafferty (Lucent) and currently being extended by Prof. Dutton's group at Stanford. The PROPHET simulator [13] uses the "dial-an-operator" methodology to construct systems of partial differential equations (PDEs) by combining existing differential operators described using a relatively simple scripting syntax. This approach does not require detailed knowledge of the discretization schemes or of the linear solver. The user does however have control over various numerical issues such as matrix conditioning, choice of linear method, etc., via the PROPHET database. The relevant parameters characterizing the physical system (e.g., material properties) can also be defined or modified "on the fly" by accessing this database. Although PROPHET's present built-in mesh generation capabilities are limited to very simple structures, meshes for more complicated geometries can be read in from an external file. This feature makes PROPHET more suitable than other application-specific software for modeling the arbitrary geometries often encountered in biological systems. 
We have used PROPHET to implement a steady-state 3-D drift-diffusion model of ion permeation through the gramicidin channel. The atomic structure of the gramicidin molecule was downloaded from the Research Collaboratory for Structural Bioinformatics Protein Data Bank [14] and input to the University of Houston Brownian Dynamics code (UHBD) [15], which generates the protein topology on a rectilinear grid. This grid information is then fed into a customized mesh generator, which outputs the computational domain in the native format required by PROPHET. Physical properties (e.g., dielectric coefficient) are assigned to the different regions of the domain and stored within the database at run-time. Several of the amino-acid residues forming the backbone of the gramicidin channel are highly polarized, and are believed to play an important role in the determining the selectivity of the channel for one species of ion over another. The effective fixed charges residing on these amino acid residues, obtained from the GROMOS molecular dynamics package [16], were used to construct a 3-D charge density distribution, which is read into PROPHET from an external file. The coupled PDEs describing the system (current continuity for the two ion species and Poisson equation) and the boundary conditions are readily constructed using existing PROPHET operators. Figure 2 shows a 2-D slice through the 3-D computational domain used to represent the gramicidin channel. The aqueous baths contains a 1.0 Molar solution of $\mathrm{NaCl}$. We show here ion flow through the gramicidin channel for two sets of conditions: (a) zero bias applied across system, and (b) $150 \mathrm{mV}$ applied bias. For these simulations we use constant ion diffusion coefficients $\mathrm{D}_{\mathrm{Na}+}=4.67 \times 10^{-11} \mathrm{~m}^{2} \mathrm{~s}^{-1}$ and $\mathrm{D}_{\mathrm{Cl}-}=1.0 \times 10^{-14} \mathrm{~m}^{2} \mathrm{~s}^{-1}$. These values were obtained from one-dimensional drift-diffusion fits to experimental data [17]. The ion mobilities are obtained from the diffusion coefficients via the Einstein relations. Experimental measurements are normally conducted by using a bias voltage in the range $0-300 \mathrm{mV}$. Figure 3 (a) illustrates the potential distribution along the channel axis at

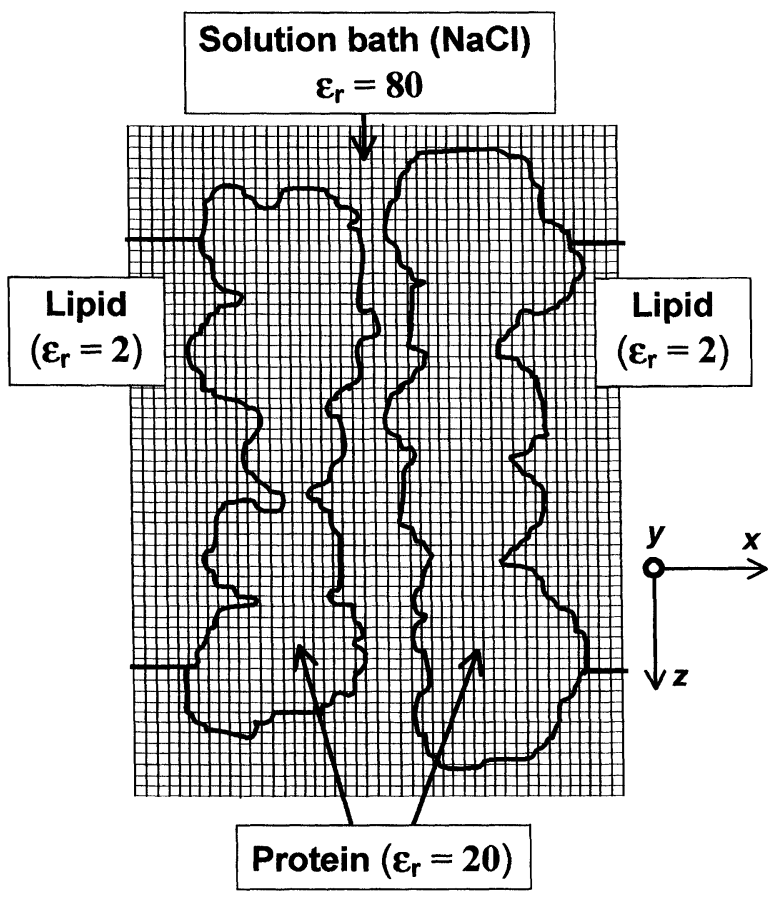

FIGURE 2 Longitudinal section $(23 \times 33 \AA)$ of the domain used for 3-D PROPHET simulation of gramicidin.

zero bias. The potential distribution for a bias of $150 \mathrm{mV}$ is shown in Figure 3(b). Figures 4(a) and (b) show the corresponding ion densities for the two cases above. The potential wells near the mouths of the channel in Figure 3(a) arise from the arrangement of fixed charges residing in the protein. Since the fixed charge is very strong, the change in ion densities under bias is somewhat subtle but noticeable. The experimentally measured current for this case is about $1.8 \mathrm{pA}$, and the preliminary PROPHET simulation gives approximately a current of $1.6 \mathrm{pA}$ when using the diffusion coefficients values above. The availability of a flexible 3-D tool should be useful now to perform detailed calibration of mobility and diffusivity models. Another issue is also to understand how the bulk mobility in the solution bath and the lower mobility in the channel should be graded in a continuum drift-diffusion approach.

Simulations were run on a Sun Ultra workstation with $1 \mathrm{~GB}$ of RAM. While 

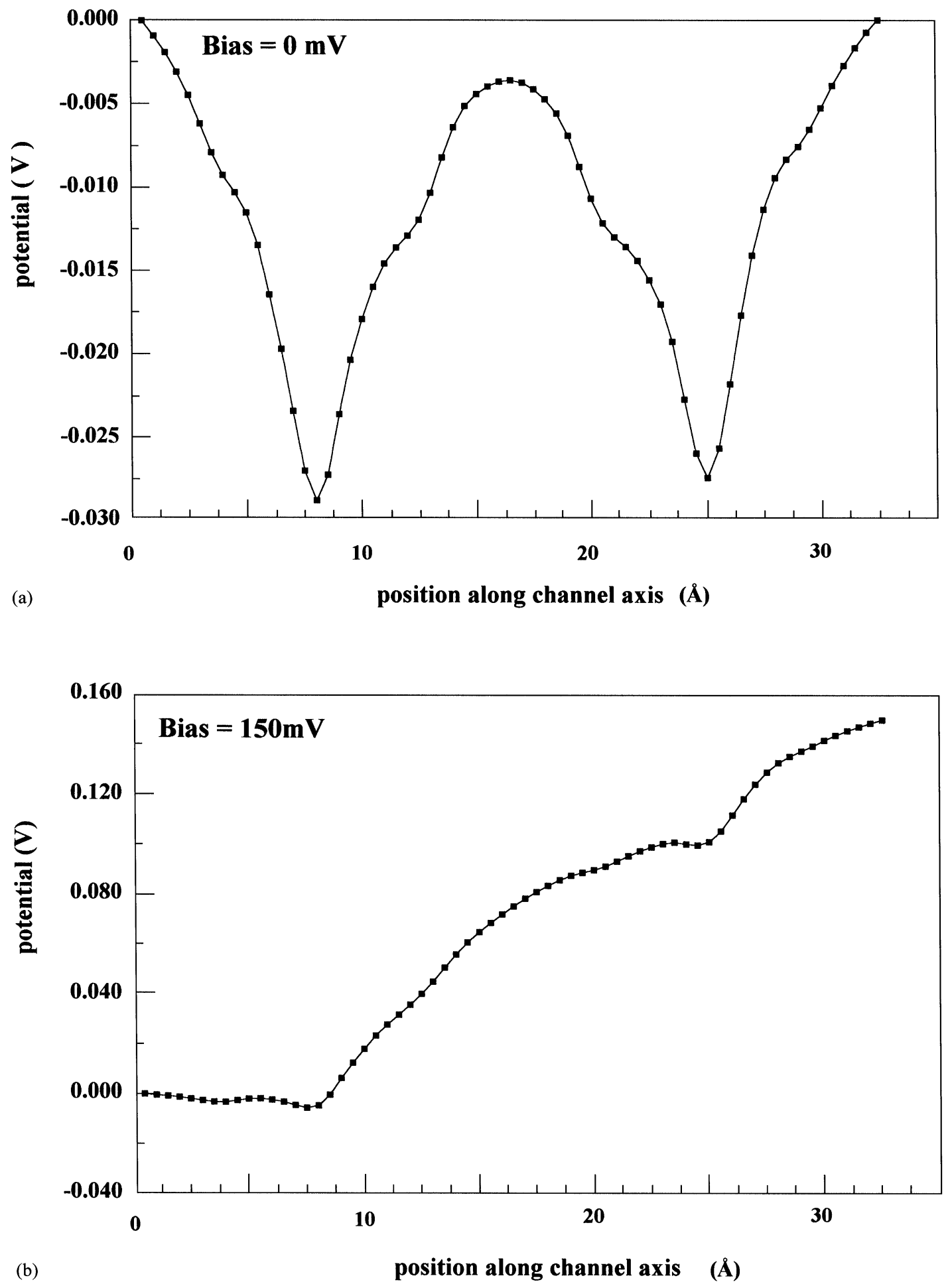

FIGURE 3 Potential distribution along the axis of the gramicidin channel with 1.0 molar solution concentration, in equilibrium (a) and with $150 \mathrm{mV}$ applied bias (b). 


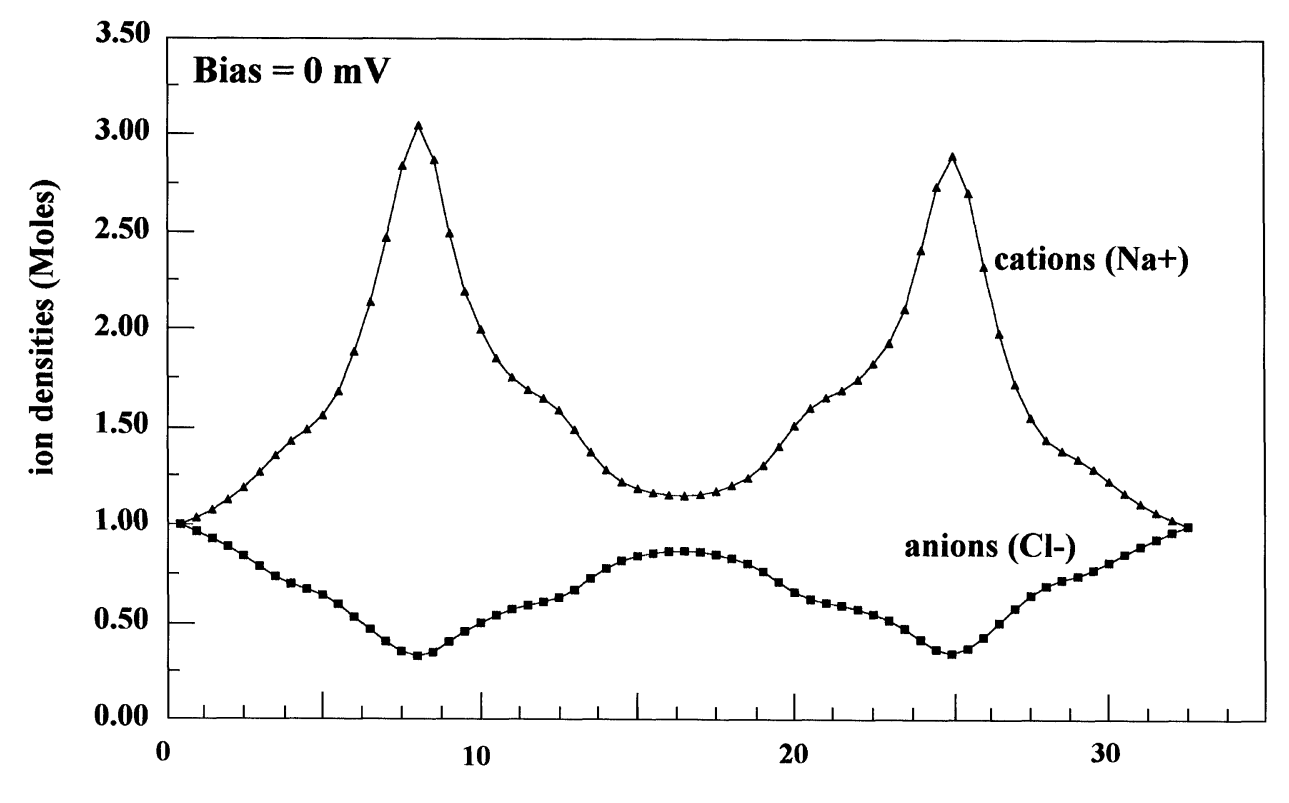

(a)

position along channel axis (Å)

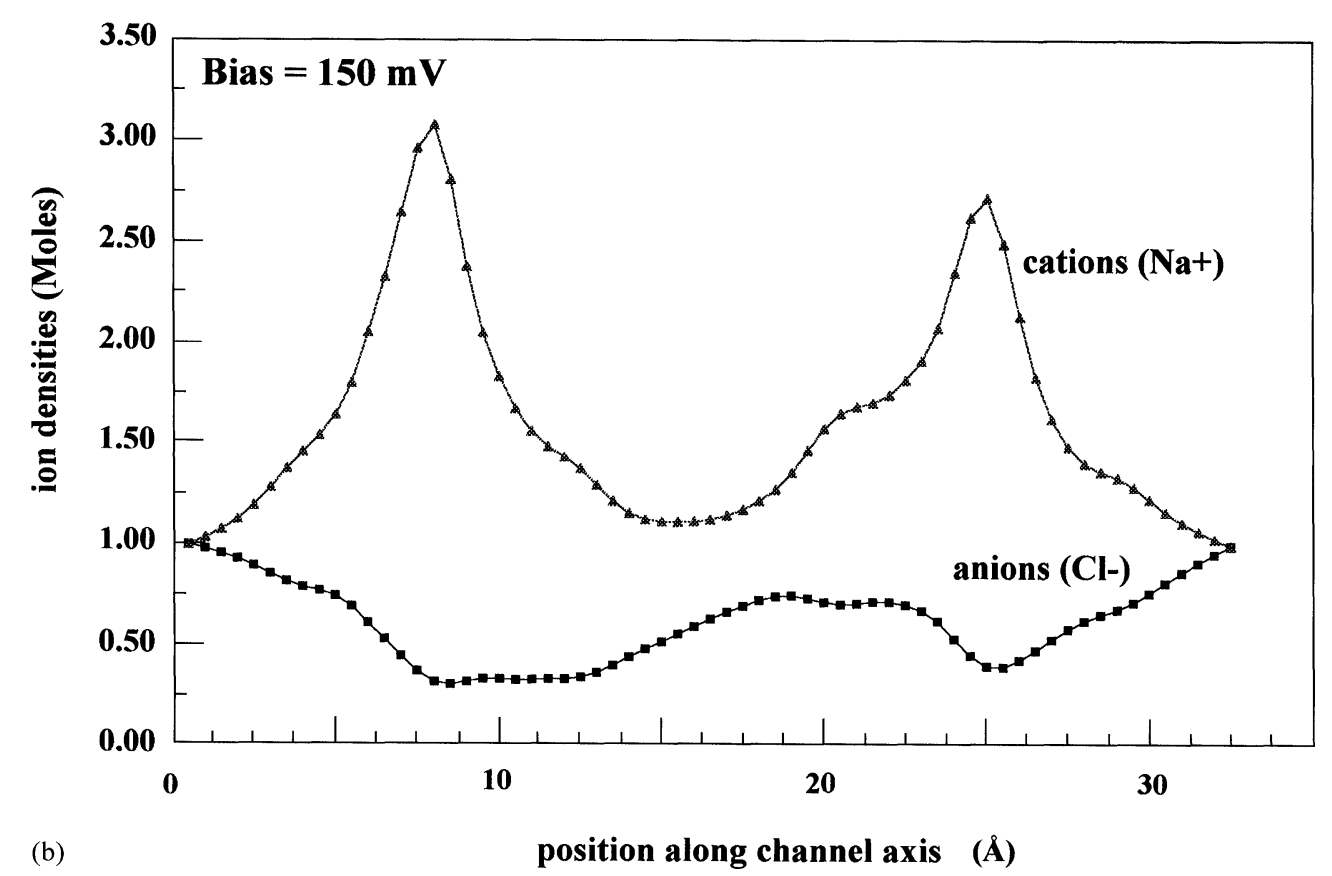

FIGURE 4 Density of $\mathrm{Na}^{+}$cations and $\mathrm{Cl}^{-}$anions along the axis of the gramicidin channel with 1.0 molar solution concentration, in equilibrium (a) and with $150 \mathrm{mV}$ applied bias (b).

executables are large, on the order $800 \mathrm{MB}$, simulations take only about ten minutes to reach steady-state. Our numerical experiments show that TCAD approaches are practical analysis tools for simulation of bio-molecular ion channels. 


\section{Acknowledgements}

This work was partially supported the NSF Distributed Center for Advanced Electronics Simulation (DesCArtES) grant ECS 98-02730, the Research Board of the University of Illinois, DARPA contract N65236-98-1-5409 (B.E.) and an NSF KDI grant to the University of Illinois. The authors thank E. Jakobsson and the staff of the Computational Biology group at the University of Illinois for discussions and help on many occasions. Also thanks to R. W. Dutton, Z. Yu and D. Yergeau of Stanford University for assistance with implementation of PROPHET applications.

\section{References}

[1] Alberts, B. (1994). Molecular Biology of the Cell, 3rd edn., New York: Garland Pub.

[2] Hille, B. (1992). Ionic Channels of Excitable Membranes, 2nd edn., Sunderland, Mass.: Sinauer Associates.
[3] Eisenberg, R. S. (1998). Contemporary Physics, 39(6), $447-466$

[4] Roux, B. and Karplus, M. (1994). Ann. Rev. Biophys. Biomol. Struct., 23, 731-761.

[5] Eisenberg, R. S. (1999). J. Membrane Biology, 171, 1-24.

[6] Hess, K. (2000). Advanced Theory of Semiconductor Devices, IEEE Press.

[7] Selberherr, S. (1984). Analysis and Simulation of Semiconductor Devices, New York, Springer-Verlag.

[8] Hockney, R. W. and Eastwood, J. W. (1981). Computer Simulation using Particles, New York, McGraw-Hill.

[9] Gross, W., Vasileka, D. and Ferry, D. K. (1999). IEEE Electron Device Letters, 20(9), 463-465.

[10] Wordelman, C. and Ravaioli, U. (2000). IEEE Transactions on Electron Devices, 47(2), 410-416.

[11] Allen, M. P. and Tildesley, D. J. (1987). Computer Simulation of Liquids, New York, Oxford.

[12] ISE-TCAD is a commercial simulation package available from ISE AG, Switzerland (http://www.ise.com).

[13] For information on PROPHET go to the site http://wwwtcad.stanford.edu/

[14] For information on the Research Collaboratory for Structural Bioinformatics Protein Data Bank go to the Web site http://www.rcsb.org/pdb/

[15] For information on the UCBD code, go to http:// mccammon.ucsd.edu/uhbd.html

[16] For information on the codes GROMOS, go to the Web site http://igc.ethz.ch/gromos/

[17] Chen, D., private communication. 

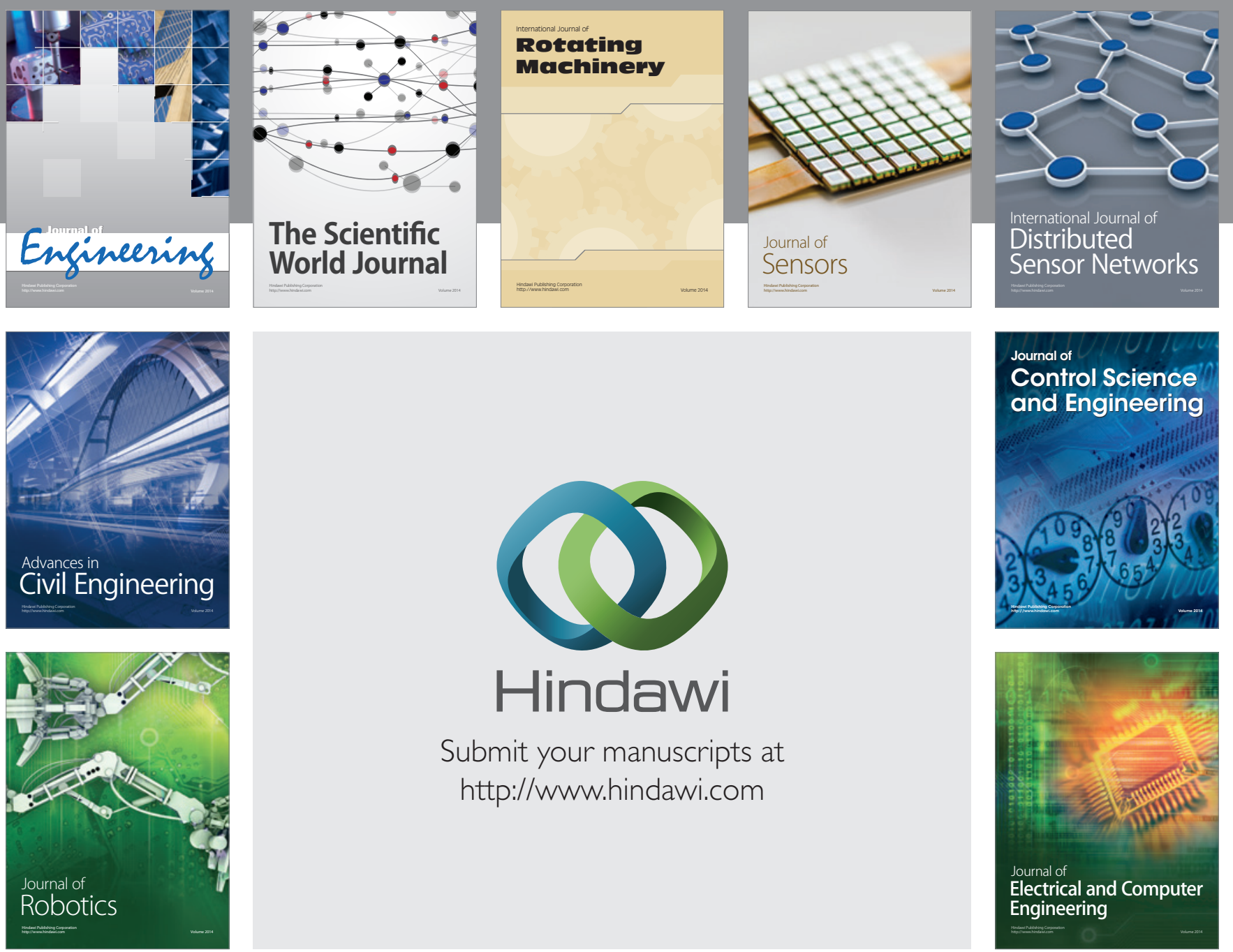

Submit your manuscripts at

http://www.hindawi.com
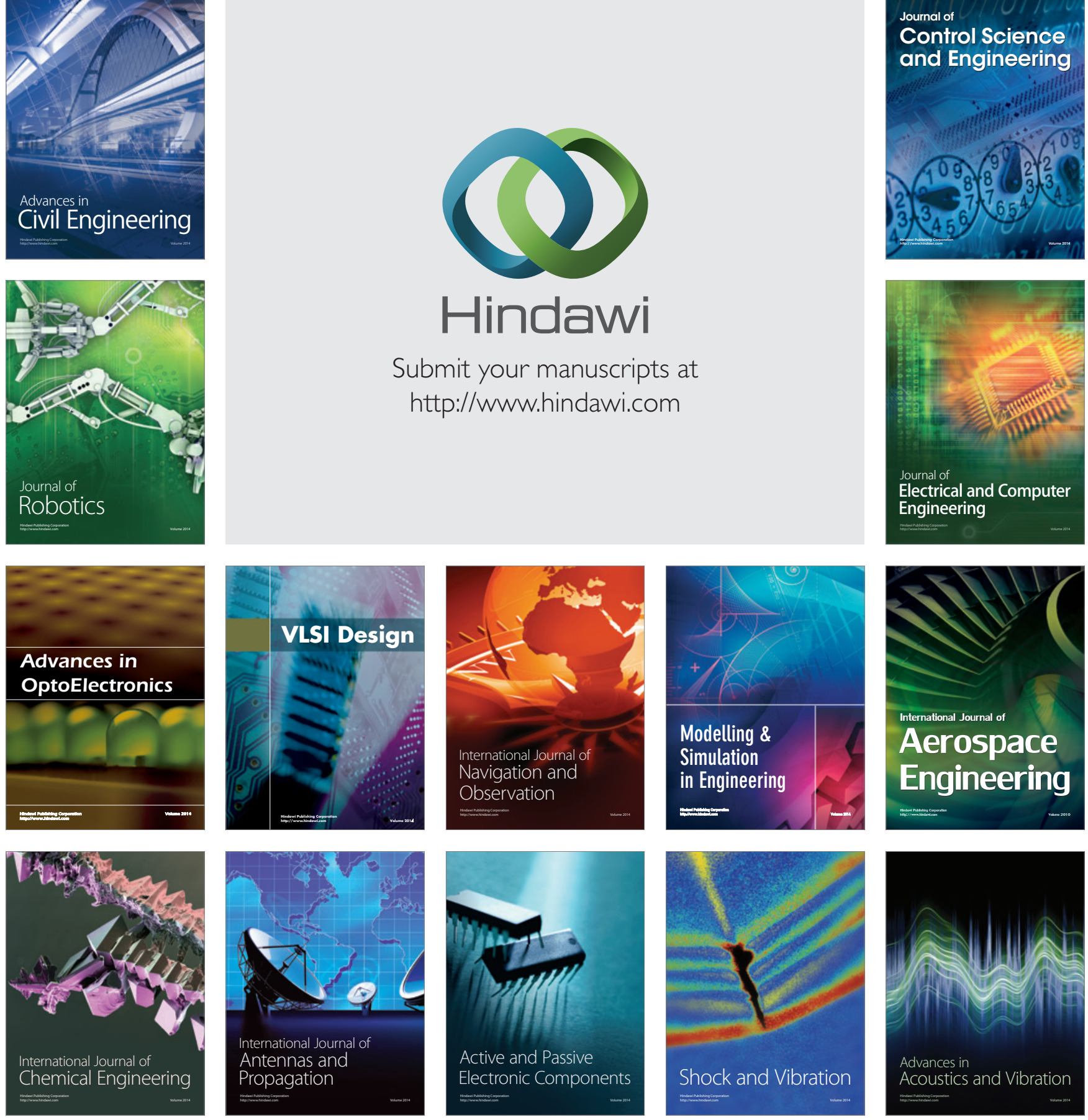